\title{
Adaptive Handoff Algorithms for Dynamic Traffic Load Distribution in 4G Mobile Networks
}

\author{
Dongwook Kim, Namgi Kim and Hyunsoo Yoon \\ Dept. of EECS, Korea Advanced Institute of Science and Technology, Daejeon, Republic of Korea \\ \{kimdw, ngkim, hyoon\}@camars.kaist.ac.kr
}

\begin{abstract}
In $4 \mathrm{G}$ mobile networks which will be a kind of packet-based cellular systems, resources are shared among all users and the amount of available resources is determined by traffic load. If traffic load is concentrated in a cell, this cell become to the hotspot cell. The botspot cell can cause to block or drop calls and consequently degrades the service quality even though available resources are remained in nejghboring cells. Therefore, it is essential to distribute traffic load of the hotspot cell in order to effectively use remained resources and maintain the acceptable service quality. We propose adaptive handoff algorithms for dynamic traffic load distribution in the hotspot cell. In the simulation, we find that these algorithms can reduce call drop rate of new and handoff calls thus enhance the service quality.
\end{abstract}

Kegwords - 4G, hotspot cell, traffic load, adaptive handoff.

\section{INTRODUCTION}

The fourth generation mobile networks which may a kind of evolution of $3 \mathrm{G}$ cellular systems aim at supporting various multimedia services over IP networks. In these networks, resources are shared among all users and the amount of available resources is determined by traffic load. The traffic load can seriously affect on QoS(Quality-of-Service) for users thus it requires efficient management in order to improve service quality.

In cellular systems, Traffic load is not uniformly distributed in service areas but can be concentrated in particular region. In a traffic accident on a highway, the accident place is crowded with people and a lot of traffic load can be generated in the cells which cover the accident place. Likewise, the cells which have heavier load than adjacent lightly loaded cells (coolspot cells) are referred to as hotspot cells. Within hotspot cells, additional call requests, new calls and handoff calls, may not be accepted because available resources are almost run out. This rejection of users' call requests can cause serious degradation of service quality. In summary, hotspot cell can be generated by sudden concentration of traffic load and this hotspot cell problem can cause poor service quality. This problem still remained as not being solved in $4 \mathrm{G}$ mobile networks

In this paper, we propose adaptive handoff algorithms as hotspot cell problem's solution which efficiently distributes traffic load of the hotspot cell to neighboring cells. Our algorithms can improve service quality by utilizing resources

This work was supported by the Korea Science and Engineering Foundation (KOSEF) through the advanced Information Technology Research Center (AITrc) and University IT Research Center Project. remained in coolspot cells. It is necessary to knowledge the amount of remained resources in coolspot cells, but resources in packet-based networks cannot be easily estimated because of packet burstiness. Therefore, we propose a novel method to accurately measure the amount of available resources.

We study related works in chapter II. In chapter III, we introduce target environments and adaptive handoff algorithms for dynamic traffic load distribution and a novel method to measure traffic load. In chapter IV, we evaluate performance of our algorithms and analyze the simulation results. Finally, we mention conclusions and future works in chapter $\mathrm{V}$.

\section{RELATED WORKS}

Many algorithms have been proposed to solve the hotspot cell problem. They are classified by resource allocation scheme and traffic load distribution scheme. In research areas of resource allocation scheme, major papers propose a centralized resource management system that allocates additional resources to the hotspot cell by borrowing them from neighboring cells. In these papers, resources are evenly allocated to the each celi and additional resources are allocated to the hotspot cell by the resource management system which control overall resources [1,2, and 3]

In research areas of traffic load distribution scheme, firstly, there are algorithms that control the transmitting power to distribute traffic load of the hotspot cell. ACS(Adaptive Cell Sizing) scheme in [4] is a representative algorithm which uses control mechanism of the transmitting power in the CDMA(Code Division Multiple Access) cellular system. In the scheme, the base station adjusts transmitting power of only those mobiles which are to be handed over to the nearest neighboring cells. It means hotspot cells shrink their service areas in order to accommodate load they can adequately handle. At this time, lightly loaded neighboring cells increase their service areas to pick up the extra traffic, which lead to use remained resources in the cells. 'In summary, through the scheme, hotspot cells can efficiently distribute traffic load and utilization of system resources is enhanced. However, the ACS scheme can be only used in the particular system and it requires the negotiation between cells in order to support seamless services for mobiles. It could not be adopted in the situation which traffic load is highly variable. 
Secondly, [5] proposes the traffic load distribution' scheme based on handoff mechanism. The algorithm, which is referred to as a soft handoff area resizing, distributes traffic load of the hotspot cell by exploiting features of the soft handoff functionality in CDMA cellular , system. The algorithm reduces the size of soft handoff area in the hotspot cell by increasing the value of the soft handoff parameter, $T$ DROP. As the size of the handoff area decreases in the hotspot cell, the mobiles in the cell boundary are forcibly handed over neighboring cells and released resources in the hotspot cell are allocated to new call requests. The scheme is based on the particular system similarly the algorithm proposed in [4]. And, if the handover call request occurred from compulsory disconnection is not handied fast, this request may be rejected even if available resources are remained.

And, in [6], traffic-driven handoff algorithms which aim at preventing the comer effect and imbalance of traffic load in the system are proposed. The corner effect means that the sudden decrease of received power due to the change from LOS(line-of-sight) to non-LOS condition when turning around corners. Proposed algorithms differently set the handoff threshold based on traffic load of cells. These algorithms are similar with our scheme in terms of traffic-driven handoff and the adaptive variation of the handoff threshold. However, the algorithms consider traffic load in order to prevent the comer effect. These algorithms cannot be solutions about the hotspot cell problem

\section{ADAPTTVE HANDOFF ALGORITHMS}

We propose two traffic load distribution schemes based on hard handoff mechanism. These algorithms spread traffic load of the hotspot cell by adaptively controlling handoff parameters, hysteresis (HYS) and the absolute threshold. The hysteresis prevents the ping-pong effect, which minimizes the number of handoffs and the absolute threshold determines the point of time to handoff. Our proposed algorithms are referred to as a simple AHL (Adaptive Handoff for Load balancing) and a dynamic AHL algorithm.

In this paper, the based architecture assumes packet-based communication systern. Therefore, all users in the same cell: share wireless resources. The information of traffic load can be obtained from the downlink broadcast channel from cells. The cell is a kind of micro cell whose diameter is from $500 \mathrm{~m}$ to $\mathrm{Ikm}$.

\section{A. Simple AHL}

Figure 1 depicts simple AHL algorithm. As shown in Figure 1, when mobile nodes move from the hotspot cell to coolspot cells, handoffs are occurred only if the hysterisis requirement is met. This mechanism can reduce traffic load of the hotspot cell fast. And, when mobile nodes enter into the hotspot cell, handoffs are occurred if both the hysteris is and the absolute threshold $\mathrm{T}_{\min }$ requirement are met. With this mechanism, remained resources in neighboring cells can be used thus utilization of system resources can be improved. It does not require a lot of changes over existing handoff procedures and does not impose high system complexity

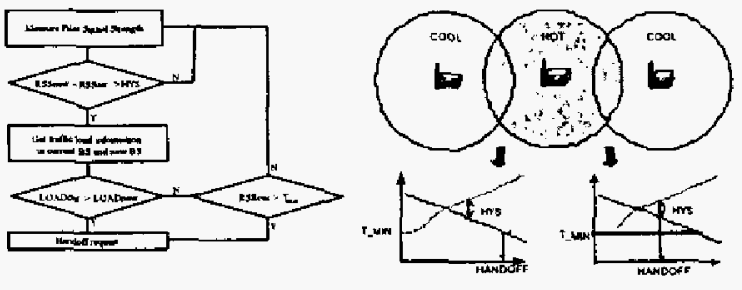

$\begin{array}{ll}\text { (a) Flowchart } & \text { (b) Concept }\end{array}$

Fig. 1, Simple AHL algorithm

B. Dynamic AHL

Simple AHL algorithm which uses the fixed threshold $\left(\mathrm{T}_{\min }\right)$ passively deals with the hotspot cell problem Therefore, we propose dynamic AHL algorithm which can distribute traffic load of the hotspot cell more dynamically than simple AHL algorithm. The dynamic AHL algorithm adaptively controls the handoff threshold according to the difference of traffic load between cells. That is, the algorithm manages the amount of traffic load of the hotspot cell by using adaptive handoff threshold. With the algorithm, traffic load between cells in the overall systems can be balanced. This algorithm is depicted in Figure 2 .

This algorithm performs handoffs when both the hysterisis and the adaptive threshold $\left(\mathrm{T}_{\text {adj }}\right.$ ) requirements are met. As shown in Figure 2, when the signal strength of the target cell is stronger by the hysteresis margin than the current cell, the mobile node enters into handoff ready state depicted in Figure 3. And the mobile sets $T_{\max }$ to the received power of this time. $T_{\text {adj }}$ is calculated by using $T_{\max }$ predefined $T_{\min }$, and the differences of traffic load. It follows Equation (1) when the traffic load's differences of the current and target cell are used linearly.

$T_{\text {adj }}=\frac{T_{\max }-T_{\min }}{2} \cdot\left|L_{C}-L_{T}\right|-\frac{T_{\max }+T_{\min }}{2}, 0 \leq L_{C}, L_{T} \leq 1$

Where, $L_{C}$ and $L_{\tau}$ are traffic load of the current and target cell, respectively.

When the difference of signal strength between current and target cell is smaller than the hysterisis in the state HANDOFF READY, then the state is returned to the state SERVE_CURRENT. In summary, the dynamic AHL algorithm can adaptively manage the amount of traffic load to solve the hotspot cell problem by balancing traffic load among cells.

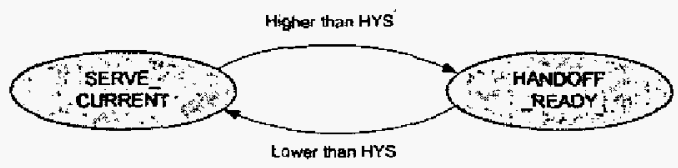

Fig. 3, State diagram in dynamic AHL algorithm 


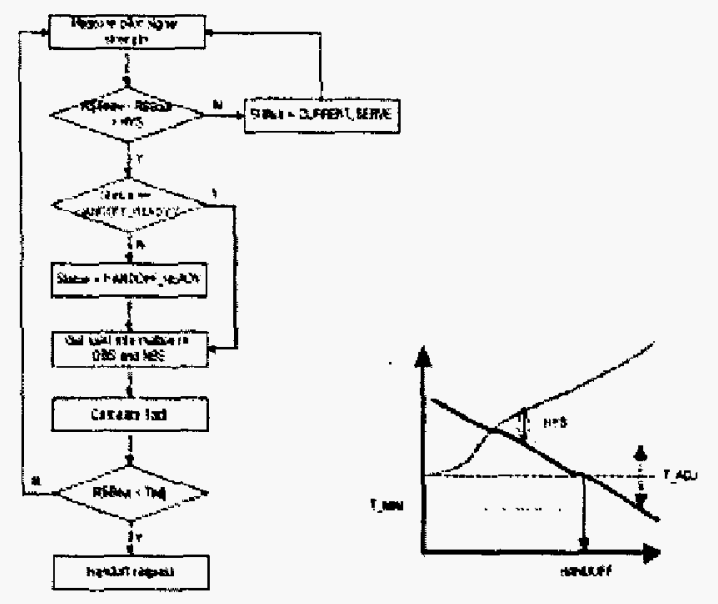

Fig. 2, Dynamic AHL algorithm

\section{Traffic Load Measurement}

It is necessary to accurately measure the amount of traffic load in order to perform our algorithms. Most of preliminary studies are based on the systems that dedicatedly use wireless resources, that is, channels. Traffic load is easily estimated as the channel occupancy rate in these systems. However, all resources are shared in packet-based communication systems. And the amount of traffic load is drastically changed due to packet burstiness. The channel occupancy rate cannot reflect the accurate amount of traffic load.

Figure 4 depicts a MAC state diagram considered in $4 \mathrm{G}$ mobile networks 7$]$. There are 5 kinds of states: NULL, ACCESS, ON, HOLD and SLEEP. Especially, in the state ON and HOLD, data is actively transmitted. But, in the state HOLD, data transmission is intermittently occurred. Hence, the state ON has both the downlink and uplink traffic channel, while the state HOLD has the downlink and thin uplink traffic channel. The other states have no traffic channels assigned. As a result, the traffic load can be estimated by measuring the number of users in the states, ON and HOLD, which is described in Equation (2).

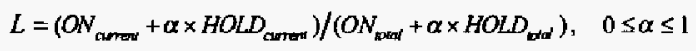

In (2), $\alpha$ is adaptive factor and the amount of traffic load $L$ varies from 0 to 1 . The value $L$ is approximated to $I$ when the current cell becomes to be the status of hotspot. And, as the number of mobile nodes is fewer, $L$ is approximated to 0 and the current cell is regarded as the lightly loaded cell.

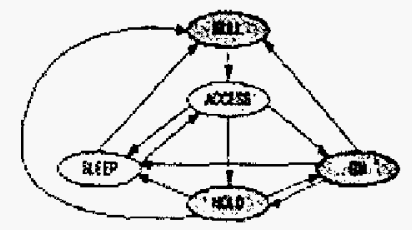

Fig. 4, MAC state diagram

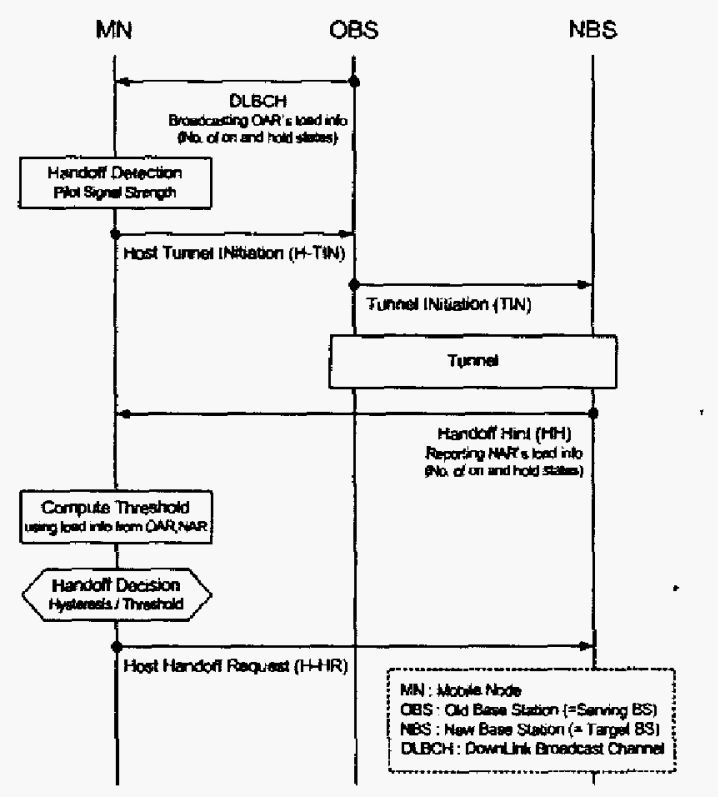

Fig. 5, Adaptive handoff procedure

\section{AHL Procedure}

Figure 5 is a generalized procedure of dynamic AHL algorithm which can be applied on $4 \mathrm{G}$ mobile networks. This handoff procedure is based on the handoff mechanism proposed in Flarion's 4G mobile networks [7]. As shown in Figure 5 , traffic load information can be periodically obtained from the downlink broadcast channel (DL.BCH). When the pilot signal strength of sorne neighboring base stations is stronger than that of the current base station plus the hysterisis, then the mobile node sends an H-TIN (Handoff-Tunnel Initiation) message to the current base station and the current base station sends TIN message to the target base station to make a tunnel in-betweens. After receiving the TIN message, the target base station directly sends a $\mathrm{HH}$ message which includes its traffic load information to the mobile node. With this procedure, each mobile node recognizes traffic load information of the current and target base station and adjusts the threshold $\mathrm{T}_{\text {adj }}$ using these load information. If the hysterisis and the threshold $T_{\text {adj }}$ satisfy the specific condition, the mobile node sends an HHR message to the target base station and performs handoff.

\section{SIMULATION RESULTS}

\section{A. The Point of Time to Handoff}

Firstly, we simulated simple AHL algorithm. The goal of this simulation is to verify the effect of the traffic distribution. In the simulation, we compared the point of time to handoff when handoffs from the hotspot cell to the coolspot cell are occurred and vice versa. Simulation parameters are shown in Table 1. This simulation was performed by Matlab [8]. Figure 6 is the result obtained after simulating 1000 times. The axis $X$ indjcates the distance from the base station $A$ to the base 
station $B$ and axis $Y$ indicates the number of handoff occurred according to the distance from the base station $\mathrm{A}$.

TABLE 1 . SIMULATION PARAMETERS

\begin{tabular}{c|c}
\hline Tx Power & $0 \mathrm{db}$ \\
\hline Cell diameter & $1000 \mathrm{~m}$ \\
\hline Distance between OBS and NBS & $2000 \mathrm{~m}$ \\
\hline Hysterisis & $10 \mathrm{db}$ \\
\hline Threshold $\left(\mathrm{T}_{\min }\right.$ ) & $-97 \mathrm{db}$ \\
\hline Simulation time & 1 hour \\
\hline
\end{tabular}

We assumed that the base station $\mathrm{A}$ is $2000 \mathrm{~m}$ far from the base station B. In case of handoffs from the hotspot cell to the coolspot cell, handoffs occurred in the point from $700 \mathrm{~m}$ to $1400 \mathrm{~m}$, on an average, about $1126 \mathrm{~m}$. In case of handoffs from the coolspot cell to the hotspot cell, handoffs occurred in the point from $800 \mathrm{~m}$ to $1600 \mathrm{~m}$, on an average, about $1217 \mathrm{~m}$. With this result, We can conclude that the simple AHL algorithm controls the point of time to handoff according to traffic load between cells thus plays a important role of traffic load distribution.

\section{B. Simulation on the Multi-cell Environment}

In the above section, we could confirm that simple AHL algorithm can distribute traffic load of hotspot cell in the two cells environment. We performed another simulation which evaluates new and handoff call drop rates in the multi-cell environment. In this simulation, we compared the simple AHL algorithm with the traditional handoff mechanism which examines only the received signal strength.

Simulation was performed on the OPNET simulator 9]. Above mentioned, we measured the drop rates of the new call and handoff call with 70 mobile nodes. In the simulation, a hotspot cell is in the center and six coolspot cells surround it. We change the available capacity of the hotspot cell from 1 to 10. The capacity of coolspot cells is fixed to 10 . The cell capacity is the number of mobile nodes which can be connected with network at the same time. Hence, the capacity of 10 means the system can provide services to 10 mobile nodes simultaneously.

\section{TABLE 2. SIMULATION ENVIRONMENTS}

\begin{tabular}{l|l|l}
\hline \multicolumn{2}{l|}{ Radio model } & General radio model \\
\hline \multirow{2}{*}{ Mobility } & Model & Random waypoint \\
\cline { 2 - 3 } & Speed & $0 \sim 30 \mathrm{~m} / \mathrm{s}$ \\
\cline { 2 - 3 } & Pause time & $0 \sim 60 \mathrm{~s}$ \\
\hline \multirow{2}{*}{ Traffic model } & $\begin{array}{l}\text { ON(10 } \mathrm{s} \text {-OFF(30s) } \\
\text { model }\end{array}$ \\
\hline Capacity & Hotspot cell & $1 \sim 10$ \\
\cline { 2 - 3 } & Other cells & 10 \\
\hline Minimum threshold $\left(\mathrm{T}_{\min }\right)$ & $-87 \mathrm{db}$ \\
\hline Normal threshold $\left(\mathrm{l}_{\text {romn }}\right)$ & $-83 \mathrm{db}$ \\
\hline \multicolumn{2}{l}{ Hysteresis } & $2 \mathrm{db}$ \\
\hline
\end{tabular}

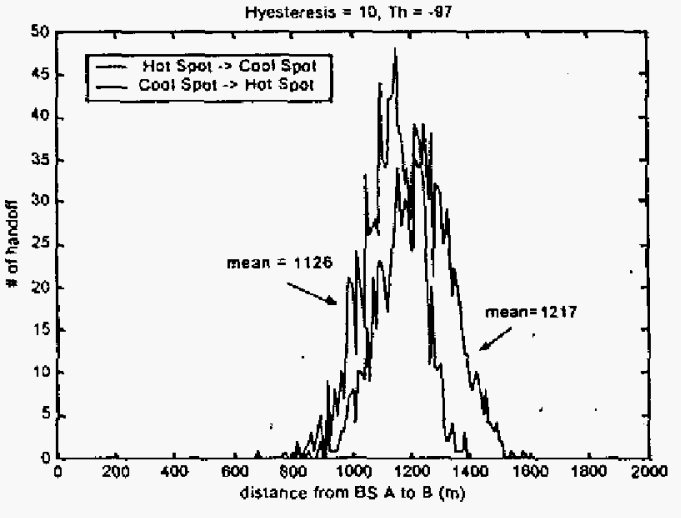

Fig. 6, Point of handoff time

If the available capacity is larger, the more mobile nodes can be connected with the network. If the available capacity of the hotspot cell is small, the hotspot cell becomes to be more congested. The specific simulation environments are shown in Table 2.

As shown in Figure 7, the total number of new calls in traditional and simple AHL algorithm is similar. However, the number of new calls in the hotspot cell of adaptive handoffs is about $21 \%$ less than that of traditional handoffs. Therefore, we can conclude that the simple AHL algorithm contributes to distributing traffic load of the hotspot cell to the neighboring coolspot cells.
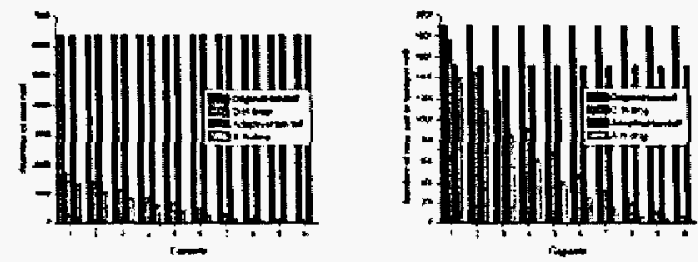

Fig. 7, Frequency of total new call and new call drop and frequency of total new call and new call drop in hotspot cell
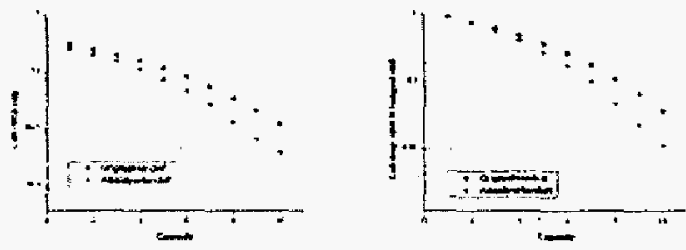

Fig. 8, Total call drop rate and call drop rate in hotspot cell
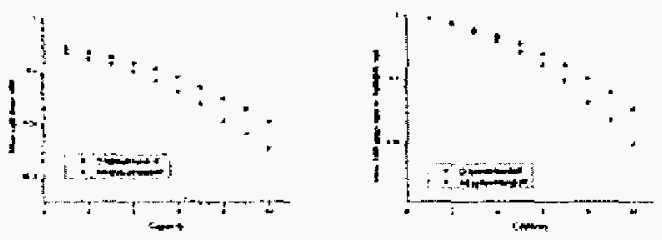

Fig. 9, Total new call drop rate and new call drop rate in hotspot cell 
Figure 8 shows that the call drop rates in simple AHL algorithm are generally less than in traditional handoff mechanism As the capacity of the hotspot cell increases, the simple AHL algorithm leads to lower call drop rates. When the capacity of the hotspot cell is less than that of the neighboring cells, the performance difference of two algorithms is not serious in spite of simple AHL algorithm's dynamic load distribution. The reason is there are not enough resources to accept mobile nodes due to the capacity limitation of the hotspot cell. Nevertheless, Due to the effect with shrinking of the hotspot cell's size, the drop rate of new calls reduces and the number of handoff calls increases as the number of new calls in the hotspot cell reduces (see Figure 12). We can find that the effect of traffic load distribution in the simple AHL algorithm becomes more significant when there are few mobile nodes in the hotspot cell or the capacity of the hotspot cell is similar with one of the neighboring cells. Therefore, we can conclude that the simple AHL algorithm contributes to enhancing the service quality.

Because the new calls in the hotspot cell reduce as the size of hotspot cell becomes smaller, the simple AHL algorithm leads to the better performance from the viewpoint of the call drop rate (sce Figure 9). And the new calls accepted in the hotspot cell are distributed into the neighboring cells as the mobile nodes move, which makes available resources to accept other calls. The decrease of the new call and the bandoff call drop rate in the hotspot cell infuence both the total new call and call drop rate (see Figure 9 and 10). That also causes the decrease of total call drop rate (see Figure 8). As the capacity of the hotspot cell grows, the number of total calls (see Figure 11) increases because the frequency of handoff per call is almost the same and the number of handoff occurred when the mobile nodes enters into the hotspot cell is invariant (see Figure 11). But, more calls can be accepted in the hotspot cell and these calls are made into another handoff calls.
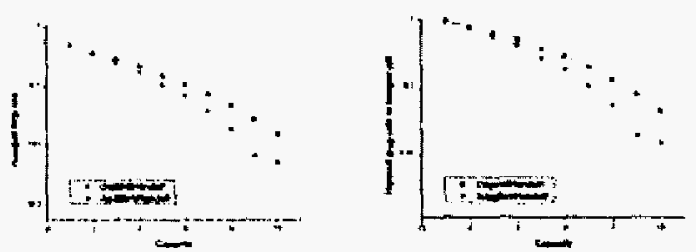

Fig. 10, Total handoff call drop rate and handoff call drop rate in hotspot cell
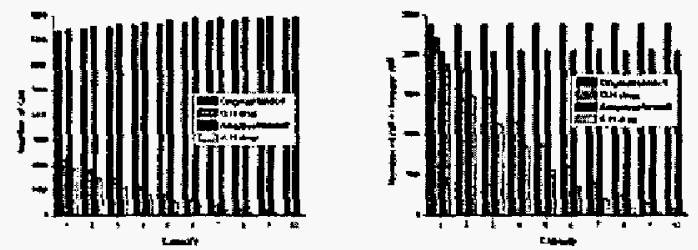

Fig. 11, Frequency of total call and call drop and frequency of total call and call drop in hotspot cell
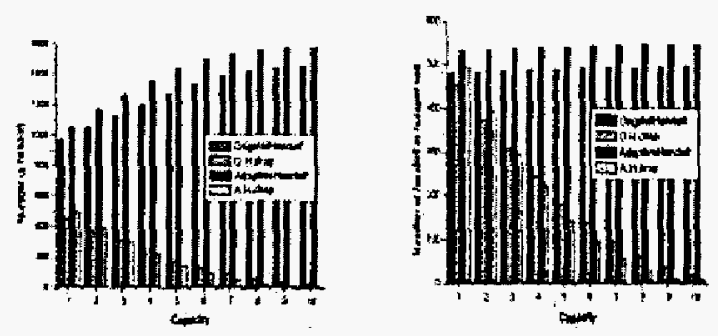

Fig. 12, Frequency of total handoff call and handoff call drop in hotspot cell

\section{CONCLUSIONS AND FUTURE WORKS}

In this paper, we proposed two adaptive handoff algorithms for the dynamic traffic load distribution and a novel traffic measurement method in $4 G$ mobile networks. The traditional handoff mechanism has shortcomings of the insufficient utilization of system resources and the degradation of service quality due to the passive traffic treatment. It may not overcome sudden traffic increases. To resolve these problems, we proposed the simple and dynamic A.HL algorithms and an effective traffic measurement method considering the packet-based cellular systems.

The two algorithms adaptively perform handoffs in accordance with traffic load for the purpose of load balancing. The simple AHL algorithm simply compares traffic load of the current cell with that of the target cell and determines the appropriate handoff method. The dynamic AHL algorithm adaptively determines the point of time to handoff according to the difference of traffic load between cells. Besides, we proposed new traffic load measurement method. It measures the traffic load by the number of users which exist in ON and HOLD state in the MAC state diagram suitable for the packet-based systerns .

To verify feasibility of proposed simple AHL algorithm, we performed the simulation which evaluates the point of time to handoff when moving between the hotspot cell and the coolspot cell. The simulation results show that movements from the hotspot cell to the coolspot cell were fast and movements from the coolspot cell to hotspot cell were slow. It means the size of hotspot cell was reduced and the size of coolspot cell was expanded, which lead to efficient distributing traffic load of hotspot cell

With multi-cell environment simulation, we also found that the simple AHL algorithm is better than traditional handoff mechanism Therefore, this algorithm enhances the service quality of users by distributing traffic load to the each cell evenly.

We have a future plan to measure the system performance considering overlapping multi-cell environment in which multiple hotspot cells exist simultaneously. Moreover, we will study a method to improve the call drop rate and the service 
quality by combining handoffs and connection admission control (CAC) schemes when a fast handoff occurs.

\section{REFERENCES}

[1] S.M. Elnoubi, R. Singh, and S.C. Gupta, "A new frequency channel assignment in high capacity mobile communication systems", IEEE Transaction Vehicular Technology, Vol. VT-31, no. 3, August 1982

[2] S. Tekinay and B. Jabbari, "Handover and chan:nel assignment in mobile cellular network", IEEE Communication Magazine, November, 1991

[3] S.K. Das, S.K. Sen, R. Jayaram and P. Agrawal, "A Distributed Load Balancing Algorithm for the Hotspot cell Problem in Cellular Mobile Networks", Proceedings, The Sixth IEEE International Symposium on High Perfomance Distributed Computing, August 1997

[4] X.H. Chen, "Adaptive traffic load shedding and its capac ity gain in CDMA cellular systems" Communications, IEE Proceedings, Vol. 142, No. 3, pp 186-192, June 1995

[5] H.G. Jeon, et al, "A channel assignment scheme for reducing call blocking rate in a DS-CDMA cellular system", IEEE 6th International Conference on Personal Commusications Record, pp. 637-641, October 1997

[6] R. Verdone and A. Zanella, "Performance of Received Power and Traffic-Driven Handover Algorithms in Urban Cellular networks", IEEE Wireless Communication, pp 60-71, February 2002

[7] S. Kapoor and J. Li, "Initial Contribution on a System Meeting MBWA", Characteristics IEEE C802.20-03/16, March 2003

[8] http:/www.mathworks.com

[9] http:/ www opnet com 